\title{
Private Practice in Psychiatry in India-Development and Seasonal Variations
}

\author{
Anil K Agarwal
}

\begin{abstract}
Sixteen years records of a private practice clinic have been analyzed. A total number of patients increased to three times while new patients increased to one and a half times. The reasons for a steady increase have been highlighted. The clinicians will need to improve communication as well as care for the needs and sensitivities of the patients. Seasonal variation was observed at the time of change of season. Lowest attendance was seen in February. There is a need to develop a database so that future clinicians could be guided properly.
\end{abstract}

Keywords: Development, India, Private practice, Psychiatry.

How to cite this article: Agarwal AK. Private Practice in Psychiatry in India-Development and Seasonal Variations. Ind J Priv Psychiatry 2018;12(1):15-19.

\section{Source of support: Nil}

Conflict of interest: None

\section{INTRODUCTION}

Mental health infrastructure is very poorly developed in India. The recent mental health survey concluded that a vast number of mentally ill do not get proper treatment and those who do get some treatment do not get it adequately. ${ }^{1}$ Psychiatric care in India is largely being provided by private practitioners as the state sector does not have enough hospitals and most of them are overcrowded. A large number of patients reach private psychiatrists and receive care from them. No postgraduate teaching program has a module that prepares psychiatrists for private practice. However, most practicing psychiatrists devise their practice style by themselves or by imitating others. There is a need of developing a module for private practice in psychiatry that could help young psychiatrists to plan their practice properly. This author has been practicing psychiatry for nearly fifty years at the same location and has been reasonably successful.The pattern of patients coming to this clinic was described earlier. ${ }^{2}$

\footnotetext{
President

Richmond Fellowship Society (I) Lucknow Branch, Lucknow, India

Corresponding Author: Anil KAgarwal, President, Richmond Fellowship Society (I) Lucknow Branch, Lucknow, India, e-mail: mradulanil@gmail.com
}

The author observed that there was a large dropout rate, and similar dropout rates were observed in different parts of India. ${ }^{3-5}$ However, after repetitive inquiries, most of the dropout patients informed that the most common reason for stopping treatment was relief in symptoms hence they did not need to consult again till symptoms relapsed.

The pattern of investigations have been studied in psychiatric patients, and it was observed that a large number of psychiatric patients underwent magnetic resonance imaging (MRI) and computed tomography (CT) scan of the head while blood investigations were few. ${ }^{6}$ The pattern of prescriptions revealed that prescriptions in the first visit were satisfactory but multiple drugs were prescribed in repeat visits. ${ }^{7}$ A multicentric study looked at the health care needs of Indian patients. Foremost needs were financial help, medical reimbursement, psychoeducation, free treatment, certification of mental illness, addressing the caregivers stress and legal aid. ${ }^{8}$ There have also been studies to find out the use of treatment guidelines in clinical practice, and it was observed that most clinicians working in the postgraduate department have read the guidelines. ${ }^{9}$ Other aspects of private psychiatric clinics like frequency of patient visit and their seasonal association have not been studied. This presentation aims at reporting the growth of a psychiatric clinic over the years and to identify any seasonal variations

\section{Description of the Clinic}

This clinic is run by a retired professor of the medical college, and he has been practicing at the same location for nearly 50 years. The clinic provides out patient department (OPD) care. The timings are from $9 \mathrm{AM}$ to 12 noon. No appointment is required and all the patients who come during this period are seen. Patients are provided a prescription, and they are free to buy medicine from stores of their choice.

Similarly, investigations can be done at any place according to their choice. These measures have been taken to reduce the fears of the patients regarding overmedication and over investigations. They should not feel that the doctor has a vested interest in selling medicines or investigations. No telephonic consultations and no emergency consultation. This clinic also does not 
have facilities for electroconvulsive therapy (ECT) and patients needing such treatments are referred to others.

\section{AIMS AND OBJECTIVES}

- To find out the number of patients that visit such a clinic over a long period.

- To find out seasonal variation in attendance if any.

\section{MATERIALS AND METHODS}

The records of patients that attended the clinic from 1st April 2002 to 31st March 2018 were screened. Yearly and monthly attendance of all patients during this period was analyzed.

\section{OBSERVATIONS}

A total number of patients seen over the years are shown in the Figure 1. The bar diagram clearly shows that the number of patients has gradually increased during this period. The increase in a number of patients is more dramatic after 2014 to 2018. The number of patients has more than doubled from 2017 to 2018. The author has reduced the number of working days in the present clinic from 2012 as he visits an Non-Governmental Organisation (NGO) twice a week and provides consultations for a charitable cause. Thus, this increase is rather remarkable. The attendance has increased to 8000 per year from 3000 to nearly 2.7 times.

The attendance of new patients (Fig. 2) has increased from 1129 to 1516 ,which is hardly a $1.5 \%$ increase. This is an important observation. This clearly shows that the number of revisits has markedly increased which reflects the patient's confidence in the clinician.
Seasonal variation: The monthly attendance did not show marked variations from month to month. The highest attendance was seen in August and January and the lowest in February and November (Fig. 3).

New patient attendance showed peak from August to November and another peak in March. There was a regular decline from November till February with a spurt in March (Fig. 4).

\section{DISCUSSION}

There is a steady increase in the number of patients attending the clinic which is more pronounced in the last four years. The clinician retired from a medical college in 1999, and the data has been taken from 2002 to nullify the effects of the position held. There does not seem to be an influence of earlier job. The number of new patients does show a steady increase. There is an increase in the number of revisits. What could be the reasons for the same? A large number of patients visiting the clinic were a second or third generation of former patients. This can be expected as quite a few psychiatric illnesses have a hereditary predisposition. It appears that the patient's faith in the clinician is an important factor in revisits. Private practice is more like a business enterprise; satisfied patients are our best ambassadors. How can one achieve the patient's satisfaction?

The patients should feel relief in their problems. A large number of psychiatric illnesses are chronic. The clinician should explain the nature of the illness and what is the likely prognosis. We should avoid false reassurances. The truth about treatment and prognosis should be told, but the timing can be according to the situation. The cost of treatment should be affordable. The patient/families

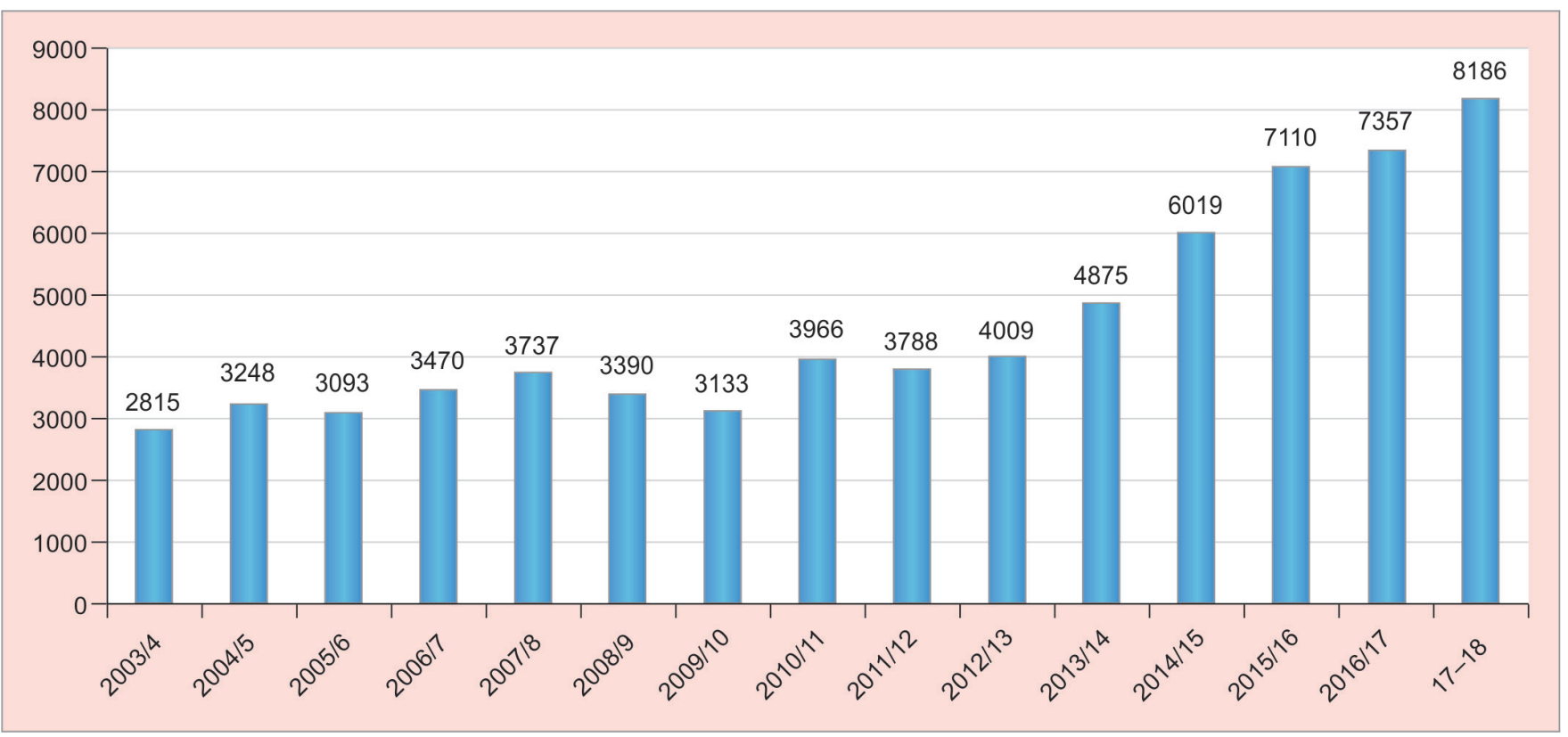

Fig. 1: Total patients attendance per year 


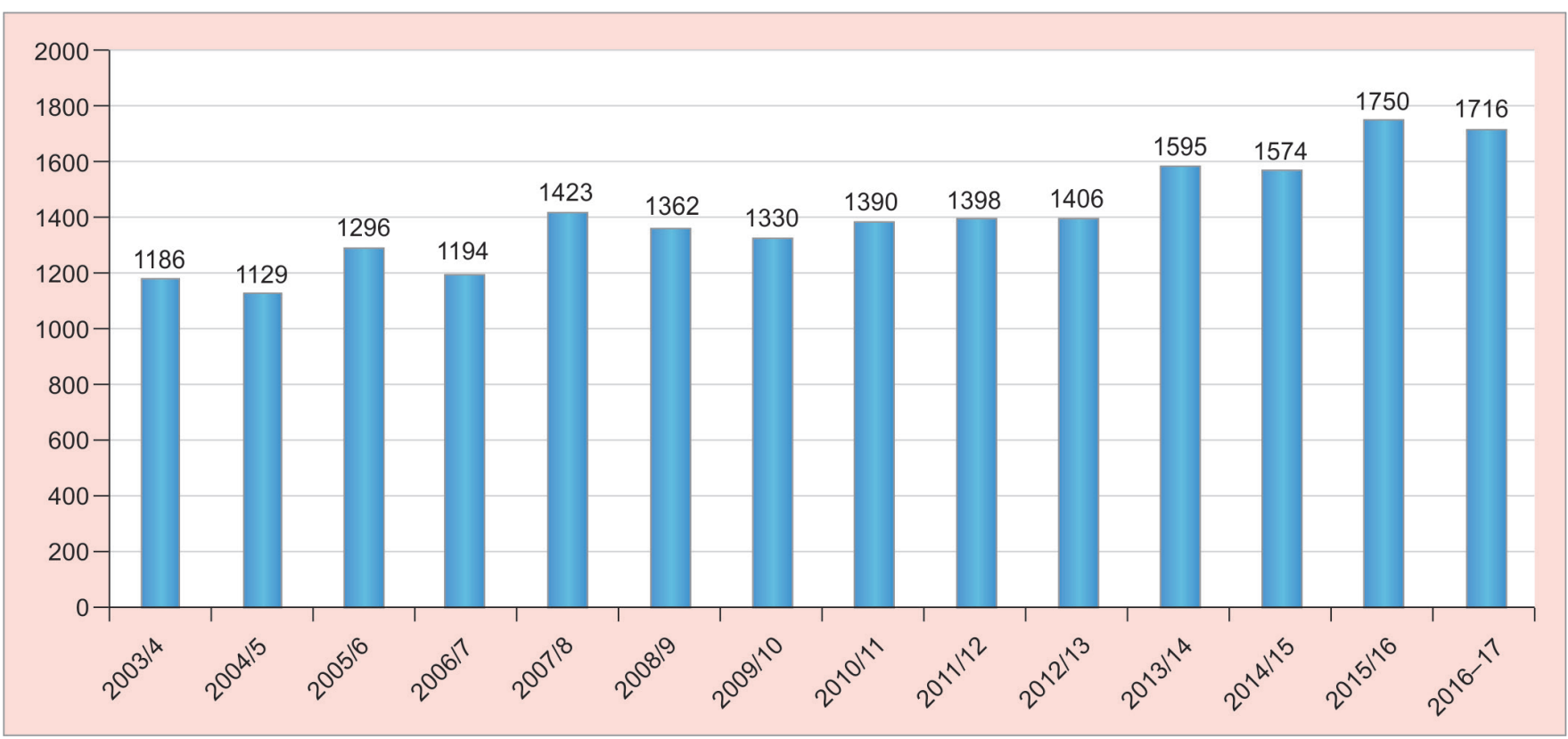

Fig. 2: New patients attendance per year

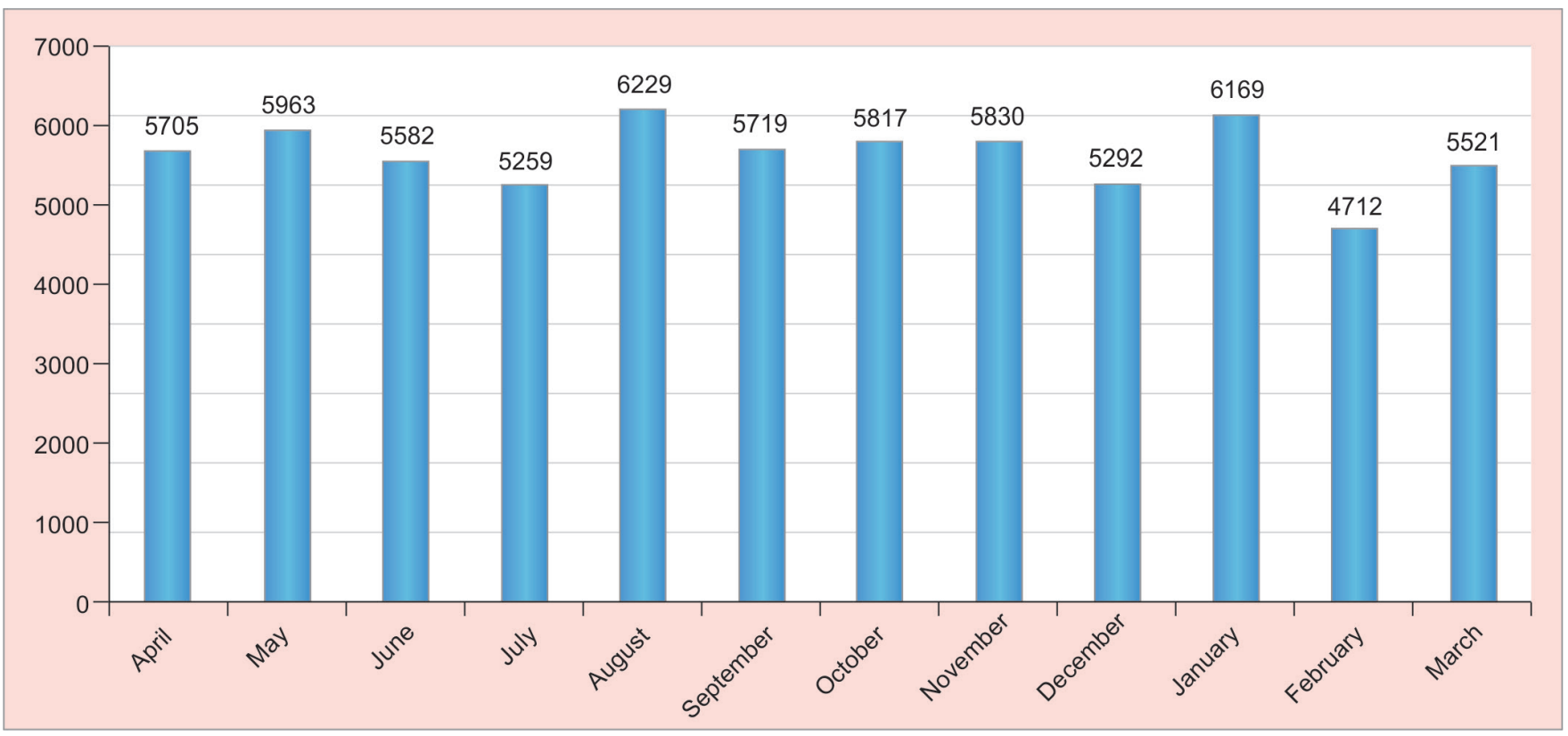

Fig. 3: Total patients attendance per month

should not feel that they are being fleeced. The general perception is that the doctors over medicate and over investigate. This clinician prescribes as few drugs as possible and unnecessary medicines like tonics, vitamins and antacids are avoided. They are prescribed when there is a definite need. The investigations are done as per specific indications. Routine investigations are avoided because many patients are not able to afford the cost of such investigations. They are given an option that routine investigations help screen other possible illnesses. I want to recall the comment of one patient who was found diabetic on the investigation and was advised to continue treatment for diabetes. The patient informed the clinician that he is taking treatment for depression as it is very painful, but he cannot afford the treatment of diabetes. CT scan head and MRI are only prescribed when there is a definite indication. More time is spent on advising patients that most mental illnesses are caused due to factors that are not picked up by these investigations. The patients are given choice to get the investigations done at places of their choice. These steps establish the credibility of the clinician.

All chronic patients are advised to take the second opinion. When the illness is chronic the patients would often seek treatment from different clinicians as well as from alternative medicine. The clinician needs to encourage the second opinion of other clinicians when the patient is in doubt. He should be able to express his opinions about alternative 


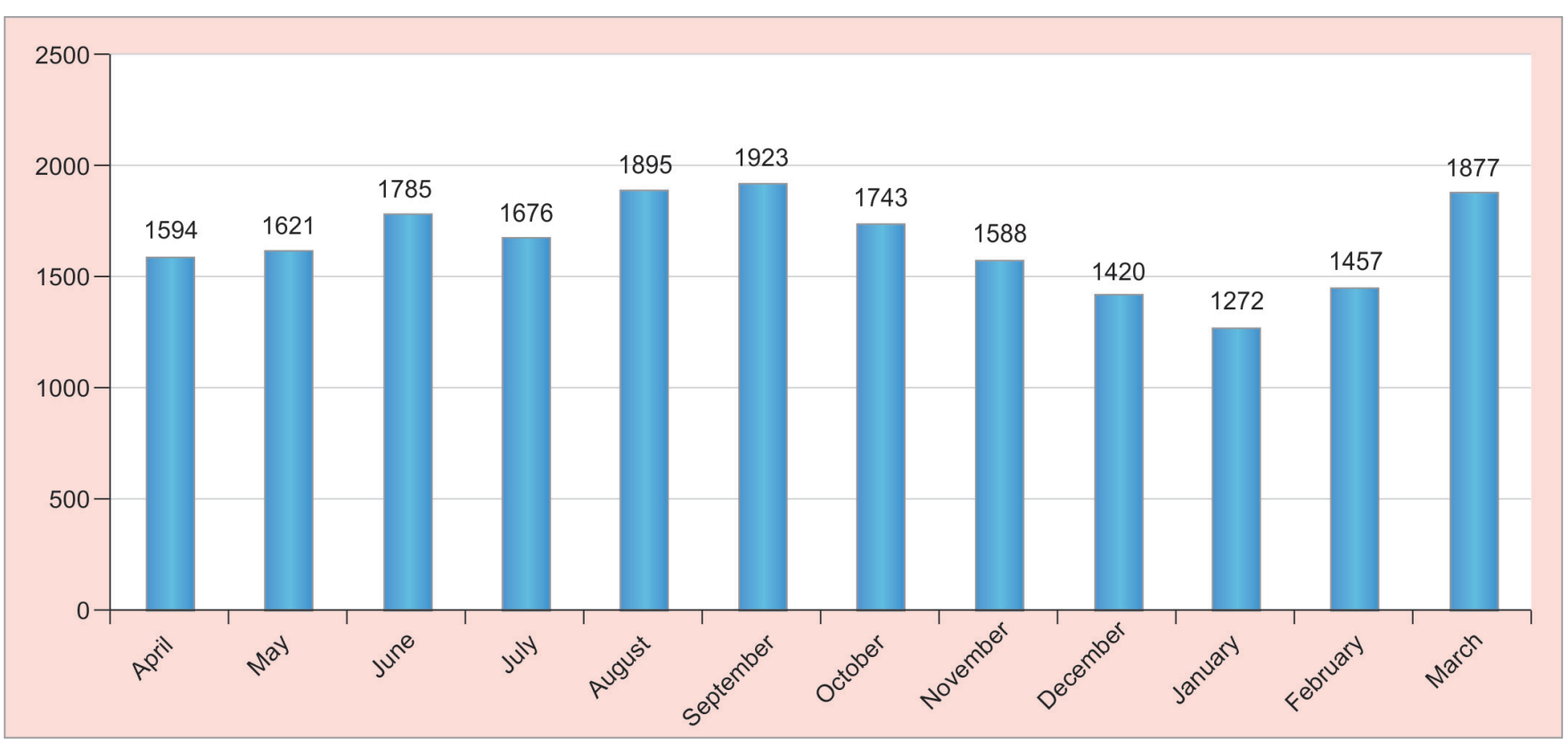

Fig. 4: New patients month-wise attendance

medicine clearly according to his belief and expertise. This author tells his patients that he does not have enough knowledge of Ayurveda and Unani medicine, but in his clinical experience he has not seen many patients obtaining relief from these medical systems in psychiatric illness. However, if a patient wishes he may try these treatments at his own risk. Many patients ask about Yoga, and this author's advice is that Yoga is good for improving mental health but cannot be a substitute for treatment. The clinician's credibility is established when a large number of patients that switched to others come back to the original clinician.

There is a large number of social issues that will determine the success of a clinician. Social and cultural beliefs of the patients are to be respected, and advice should follow the general cultural norms. It does not mean that we endorse faith healing practices or encourage cultural practices like gender discrimination. The care taker's problems and views are to be respected and their problems seriously viewed.

Looking at the seasonal variation one can notice that there are two peaks, one is from August to October and the second in March. February seems to be the lowest attendance month. These variations could be due to multiple factors. Some of these could be due to clinical reasons and others due to psychosocial factors.

There is a belief that mental illnesses are related to the season. There is a clear association of some affective disorders to be precipitated at the time of change in season. March to April and September to October are the months when seasons change in this part of the country. Stresses of examinations may precipitate some illnesses during the examination period, i.e., March to April.
Psychosocial factors like harvesting season, religious festivals and availability of money may affect consultation seeking behavior. These aspects can be better teased out once we do a more detailed analysis of clinical and social parameters.

\section{CONCLUSION}

This paper presents the annual and monthly attendance of a private psychiatric clinic in Northern India. The data may not be entirely applicable for all clinics as every clinic is given different meaning by the public. However, some generalities can be drawn regarding the steady development of the clinic over a period. Methods used by the author to develop the confidence of the patients and families have been highlighted. There is a need to develop a database so that a course in private practice could be introduced in the postgraduate training program.

\section{REFERENCES}

1. National mental health survey,2015-16, Ministry of Health and Family Welfare, Government of India. Pub. National Institute of Mental Health and Neurosciences: 2016-23-24.

2. Agarwal AK. Analysis of patients attending a private psychiatric clinic. Indian J Psychiatry 2012;54:356-358.

3. Grover S, Dua D, Chakrabarti S, Avasthi A. Dropout rates and factors associated with dropout from treatment among elderly people attending the outpatient service of a tertiary care hospital. Indian J Psychiatry 2018;60:49-55.

4. Devnathan S. Personal communication. 2010.

5. SureshKumar PN. Pathways to psychiatric care; An Experience in Kerala. Indian J Private Psychiatry 2016;10:22-27.

6. Gupta DK. Suthar N, Singh V, et.al Frequency and Pattern of Radiological and Laboratory investigations in patients with mental illnesses: A Study from North Rajasthan. Indian J Psychiatry 2016;58:183-189. 
7. Grover S, AvasthiA,Sinha V. et.al. Indian Psychiatric Society Multicentric Study: Correlates of prescription Patterns of Psychotropic in India. Indian J Psychiatry 2016;58:417-424.

8. Grover S, Avasthi A, Nebhinani N. et.al. Indian Psychiatric Society-Multicentric Study on assessment of healthcare needs of patients with severe mental illness. Indian J Psychiatry 2015; 57:43-50

9. Grover S, Avasthi A, Views about clinical practice guidelines of Indian Psychiatric Society: A survey of Psychiatrists in India. Indian J Psychiatry2009;51:127-133. 